\title{
Obstacle-avoiding Rectilinear Steiner Tree Construction
}

\author{
Liang Li and Evangeline F. Y. Young \\ Department of Computer Science and Engineering \\ The Chinese University of Hong Kong \\ Email: \{1li,fyyoung\}@cse.cuhk.edu.hk
}

\begin{abstract}
In today's VLSI designs, there can be many blockages in a routing region. The obstacle-avoiding rectilinear Steiner minimum tree (OARSMT) problem has become an important problem in the physical design stage of VLSI circuits. This problem has attracted a lot of attentions in research and several approaches have been proposed to solve this problem effectively. In this paper, we will present a heuristic maze routing based approach to solve this OARSMT problem. It is commonly believed that maze routing based approaches can only handle small scale problems and there is a lack of an effective multi-terminal variant to handle multi-pin nets in practice. We will show in this paper that maze routing based approaches can also handle large scale OARSMT problems effectively. Our approach is based on the searching process as in maze routing and can handle multi-pin nets very well in both solution quality, running time and memory space usage. We have compared our results with those of the previous works and can show that we can out-perform the best previous results on this problem [15] by giving an OARSMT with $2.01 \%$ less wire length on average and can make a $27.04 \%$ improvement in wire length in comparison with a lower bound of the optimal solution on average, while the running times are all very short and comparable to those in [15]. Besides, due to the flexibility of maze routing, we can handle different kinds of obstacles with different convex or concave rectilinear shapes directly without a need to partition each blockage into a set of rectangular sub-blockages, which will increase the size of the problem.
\end{abstract}

\section{INTRODUCTION}

Construction of rectilinear Steiner minimum tree (RSMT) is an important problem in VLSI physical design. It is useful for the detailed and global routing steps, and is important for congestion, wire length and timing estimations during the floorplanning or placement step. This classical problem has long been shown to be NP-complete and has attracted a lot of attentions in research. The original RSMT problem assumes no obstacles in the routing region. In today's VLSI designs, there can be many routing blockages however, like macro cells, IP blocks and pre-routed nets. Therefore, the RSMT problem with blockages, called obstacle avoiding RSMT (OARSMT), has become an important problem in practice and is an interesting problem theoretically. Since the RSMT problem is NP-complete, the OARSMT problem is also NP-complete as it is a generalized version of RSMT.

Clarkson et al.[1] considered only 2-pin nets and presented an $O\left(n(\lg n)^{2}\right)$ time algorithm to compute a rectilinear shortest path between two pins avoiding polygonal obstacles, where $n$ is the number of pins and obstacle boundaries. Wu et al.[2] introduced a sparse connection graph, called track graph, to reduce the search space for computing the shortest path between two points avoiding obstacles. Ganley et al.[3] proposed an algorithm to construct an optimal 3-terminal or 4-terminal OARSMT. Heuristics, called G3S, G4S and B3S, were developed for the cases with less than 20 terminals. Zheng et al.[4] proposed an algorithm to find obstacleavoiding shortest paths using an implicit connection graph approach. Yang et al.[5] presented a complicated 4-step heuristic to the OARSMT problem and their approach works well when the terminal number is less than seven and when the obstacles are convex.

More recently, $\mathrm{Hu}$ et al.[6] developed an efficient hierarchical heuristic called FORst, that partitions all pins into subsets, then connects pins in each subset, and finally constructs an OARSMT using a connection graph [7] based approach. Their method can tackle large scale problems efficiently. Based on an ant colony optimization, Hu et al.[8] proposed another non-deterministic local search heuristic, called An-OARSMan, to handle small-scale OARSMT problems with complex obstacles of both concave and convex shapes. This approach can achieve shorter wire length than FORst when the number of terminals is less than 100. Although An-OARSMan is flexible in handling complex obstacles, it takes extremely long running time for large scale designs. CDCTree proposed by Shi et al.[11] is based on a current driven circuit model and it can achieve shorter wire length than An-OARSMan. Shen et al.[9] proposed a connection graph based approach to solve the OARSMT problem, using the spanning graph in [10] which did not consider obstacles. In their approach, an obstacle-avoiding spanning graph is first constructed and then transformed into an OARSMT. Feng et al.[12] proposed a method to construct obstacle-avoiding Steiner tree in an arbitrary $\lambda$-geometry by Delaunay triangulation. Its running time complexity is just $O(n \log n)$, where $n$ is the total number of terminals and obstacles.

Very recently, Wu et al.[13] presented another algorithm for constructing OARSMTs. Their approach will first find a minimum spanning tree of all the terminals by applying a partitioning method. The segments intersecting with the obstacles will be removed, forming a set of sub-trees. An ant colony optimization based approach is then used to connect the sub-trees back into a single tree which is rectilinearized at the end to given an OARSMT. Hentschke et al.[14] presented AMAZE, a fast maze routing based algorithm to build steiner trees. A sharing factor and a path-length factor were introduced to trade-off wire length for delay. However, when dealing with multiple paths, the algorithm adopts traditional ways of selecting just one path from multiple paths with a biasing technique. The most recent work on this problem is by Lin et al.[15]. They extended the connection graph based approach in [9] by identifying many "essential" edges which can lead to more desirable solutions in the construction of the obstacle-avoiding spanning graph. They have also improved the OARSMT transformation procedure in [9] significantly, and develop an effective refinement scheme for the U-shaped connections in an OARSMT to further reduce the total wire length. The result obtained by this approach is the most updated one among all the others.

It is commonly believed that the maze routing based approaches are suitable for small scale problems only. The major drawback is the lack of a multi-terminal variant to handle multi-pin nets. Besides, its time complexity and memory usage can grow very large as the routing area expands. In this paper, we will show that maze routing based approaches can also handle large scale OARSMT problems effectively. In our algorithm, to handle multi-pin nets, multiple paths between the pins are kept until all the pins are reached, then a MST based method is used to select between those paths to create an OARSMT. A post-processing step is then performed to further reduce the total wire length. The basic maze routing step is implemented 
efficiently by using a heap data structure and by propagating on the simplified Hanan grid which is formed by the original Hanan grid with all the intersection points lying inside an obstacle deleted. This heap data structure has also helped to handle multi-pin nets very efficiently. Experimental results show that we can out-perform the most updated previous work [15] on this problem in terms of wire length in a very short running time. We can achieve $2.01 \%$ less wire length on average and a $27.04 \%$ improvement in comparison with a lower bound of the optimal wire length, while the running times are all very short and comparable to those in [15].

In this paper, we will first formulate the problem in section II. Our maze routing based approach will be presented in section III. Experimental results will be shown in section IV before the conclusion in section V.

\section{Problem Definition}

Definition 1: OARSMT Problem: Let $B=\left\{b_{1}, b_{2}, \ldots, b_{k}\right\}$ be a set of non-overlapping rectilinear blockages in a 2-dimensional space $R$. Let $P=\left\{p_{1}, p_{2}, \ldots, p_{m}\right\}$ be a set of pins for a $m$-pin net in $R$ such that no pins $p_{i} \in P$ lie inside an obstacle $b_{j} \in B$. Construct a rectilinear Steiner minimum tree connecting all the pins in $P$ to achieve a minimum total length (measured by Manhattan distance), while avoiding intersection with any blockages in $R$.

\section{A. Properties of OARSMT}

In the OARSMT problem, a pin cannot lie inside an obstacle but it can be at the corner or on the boundary of an obstacle. An obstacle cannot overlap with another obstacle, but it can point-touch an obstacle at a corner or line-touch at a boundary. The edge of the OARSMT cannot intersect with a blockage, but it can run along the borders of the obstacles.

\section{OUR APPROACH}

Our heuristic approach is based on the maze routing method, but our maze routing engine can handle directly multi-pin nets effectively. The efficiency of this routing engine can be further improved by the use of a heap data structure and by propagation on the simplified Hanan grid. By using this multi-pin maze routing approach alone, we can already obtain results better than those generated by the most updated previous work [15]. Then, a fast post-processing step will be applied to scan the paths connected to each pin once to further minimize the total wire length. In the following, we will first explain the general approach we employed to handle multi-pin nets in our maze routing engine. Details of the implementation will be explained in the next sub-section, followed by a description of the post-processing step at the end.

\section{A. Handling of Multi-pin Nets}

Given a $m$-pin net $P=\left\{p_{1}, p_{2}, \ldots, p_{m}\right\}$, we will pick one pin to start with. In our algorithm, we will choose the pin whose position is the nearest to the boundary. Assume that $p_{\pi(1)}$ is selected as the first pin. Then, we will propagate from $p_{\pi(1)}$ to find the second pin to connect with. Let $p_{\pi(2)}$ be the second pin we found, i.e., $p_{\pi(2)}$ has the shortest Manhattan distance from $p_{\pi(1)}$ in comparison with others. Note that multiple paths of this length will be found and recorded in our maze router. Then, we will propogate from all these paths to find the third pin, again, recording multiple paths of the same shortest Manhattan length to reach this third pin. This process is repeated until all the pins in $P$ are reached. After this process, we will find all the Steiner points lying on the paths found and record the Manhattan distances between the points (pins and Steiner points). From this information, we will perform once a minimum spanning tree algorithm to connect all the points, followed by a garbage collection step to remove dangling connections which are connected to Steiner points only. An example illustrating our approach to handle multiple pins is shown in Fig. 1. The pseudocode is described as follows.

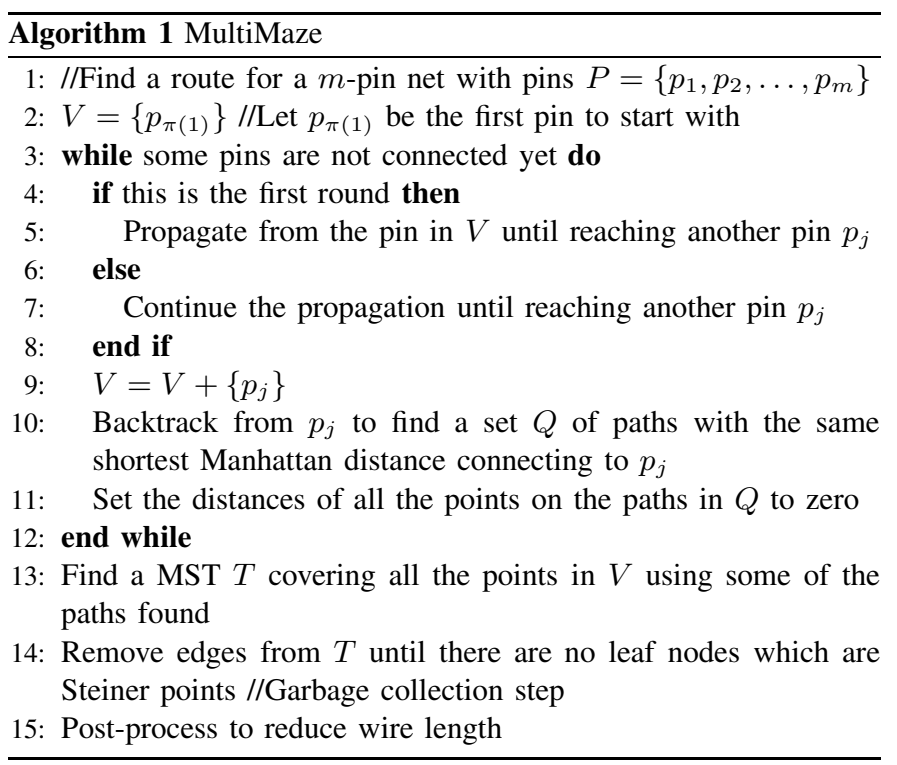

Our approach is different from traditional maze routing based methods in handling multi-pin nets. Instead of connecting the pins sequentially and decide on the paths once some new pins are reached, multiple paths are kept and path selection is delayed until all the pins are reached and the final route is constructed globally by finding an MST. In order to route fast, the propagation is performed on the simplified Hanan grid and a heap data structure is used such that the current shortest path will always be expanded first. One important technique in our approach to shorten the running time is that after some new paths are found and the distances of the points on them are set to zero, instead of starting the maze routing once again from scratch, we will continue the propagation from the last round. The heap data structure allows us to update the shortest distances at those affected points fast without repeating works which have been done before. We will explain in details the propagation step, the backtracking step and the final post-processing step in the following sub-sections.

\section{B. Propagation}

We can see from the pseudocode of MultiMaze that in each round of the propagation process (line 3 to 12), the backtracking step will be performed once and the backtracking result will affect the propagation in the next round. In this section, we will focus on the propagation process, while the backtracking step will be described in the next section. The pseudocode of the propagation process is shown in Algorithm 2.

In the propagation process, a heap $H$ is maintained. This heap will store 2-tuples $((x, y), d)$ where $(x, y)$ is the coordinate of a point and $d$ is the distance of a path that can reach $(x, y)$ from some connected points. The heap $H$ will be sorted according to the $d$ values. A 2dimensional array $\operatorname{dist}[][$ ] keeps the shortest distance found so far from a connected point and the value $\operatorname{dist}[x][y]$ will be zero if the point $(x, y)$ is connected. The while-loop on line 10 repeats while $\min (H)<$ best where $\min (H)$ is the shortest distance stored in 


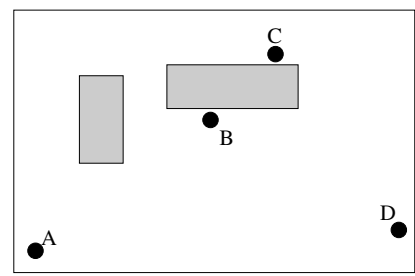

(a) Four pins to connect.

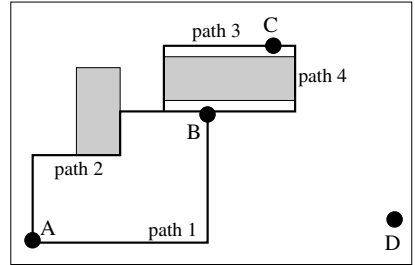

(c) Connect to pin C. Form two more paths.

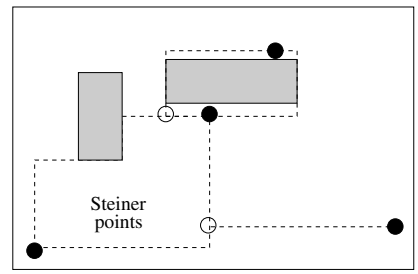

(e) Steiners points and path lengths are found.

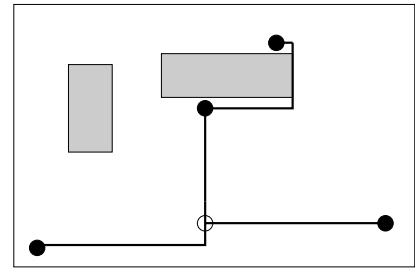

(g) After garbage collection step.

Fig. 1. An example illustrating our approach

$H$ and best is the shortest distance found so far. This is to ensure that all the paths of the same shortest Manhattan distance connecting to the next pin $p_{j}$ will be taken out from the heap and processed. Note that nothing is done if the distance stored on the heap top is larger than the distance recorded in $\operatorname{dist}[x][y]$ (line 12), since in this case, we had already found a shorter path reaching $(x, y)$ in some previous round and no updates are needed. An important step in our approach to improve the efficiency is that in each round of the outer while-loop (line 6), some new paths are found and the dist [][ ] value of all the points on these paths will be set to zero for the next around, and instead of starting the maze routing once again from scratch, we can continue with the propagation from the last round after inserting some new elements into $H$. The heap data structure allows us to update the distances of the affected points rapidly without repeating works which have already been done. Imagine a point whose distance value is independent of the newly found paths, its information in $H$ and $\operatorname{dist}[][$ ] is kept and will be useful, while for those points that can get a shorter distance because of the newly found paths, their information in $\operatorname{dist}[][]$ will be updated automatically after inserting the 2-tuples $((x, y), 0)$ into $H$ (line 8$)$ for all the points $(x, y)$ lying on those newly found paths. A simple example is shown in Fig. 2 to illustrate this propagation process.

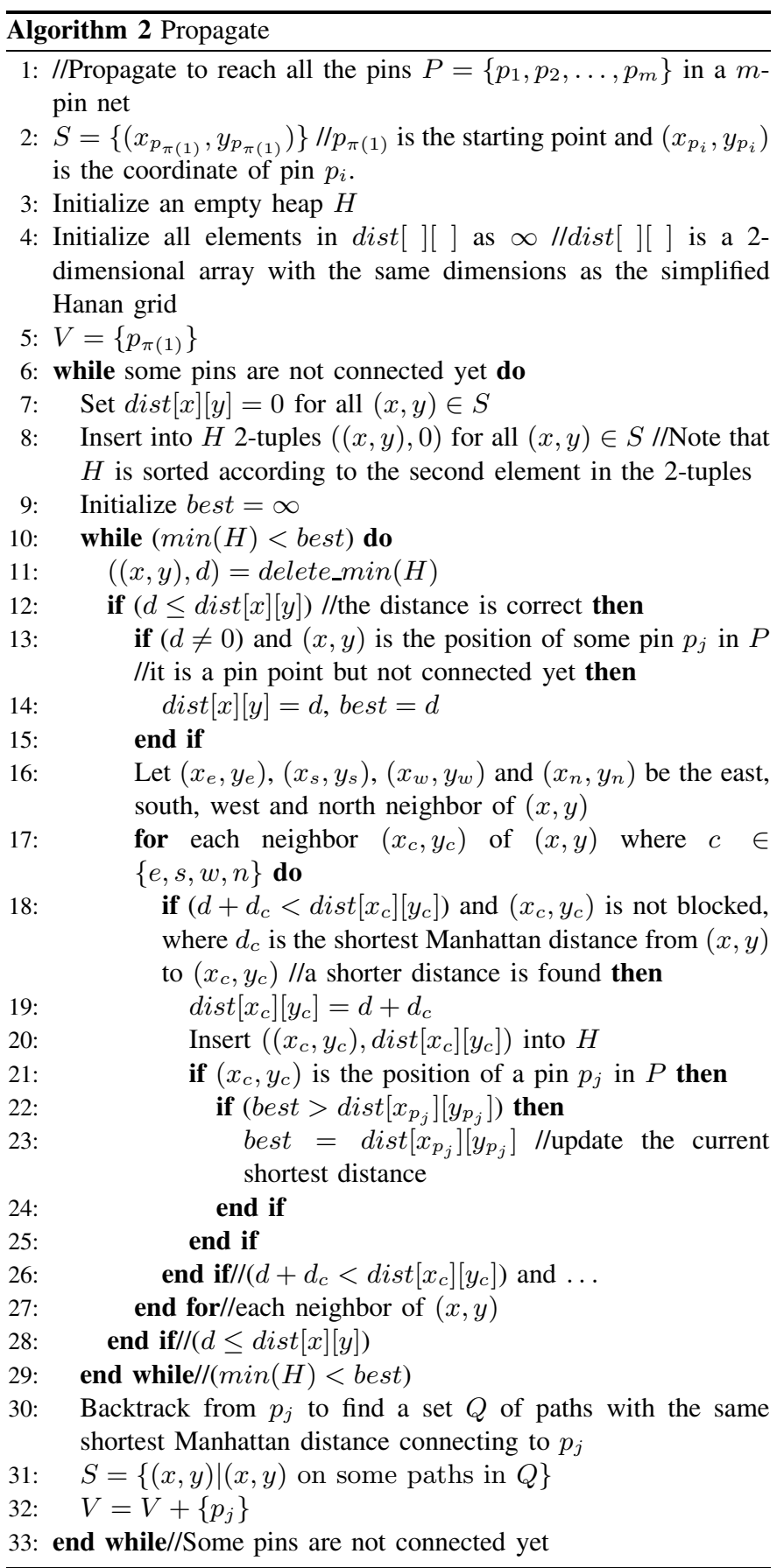

\section{Backtrack}

After a next pin $p_{j}$ is found by propagation, backtracking will be performed to find all the paths connecting to $p_{j}$ with the same shortest Manhattan distance. Basically, our backtracking strategy will try to move without turning as much as possible, unless meeting an obstacle, or seeing an obstacle on one side of the path. At the end, we will find multiple paths of the same length, and the paths found will be "moving around" the obstacles. An example is shown in Fig. 3. The pseudocode of this backtracking step is shown in Algorithm 3.

In the pseudocode of Backtrack, a stack $K$ is used to keep all the possible branching points on the paths (points at which a path can change in direction to form another feasible path). The elements 


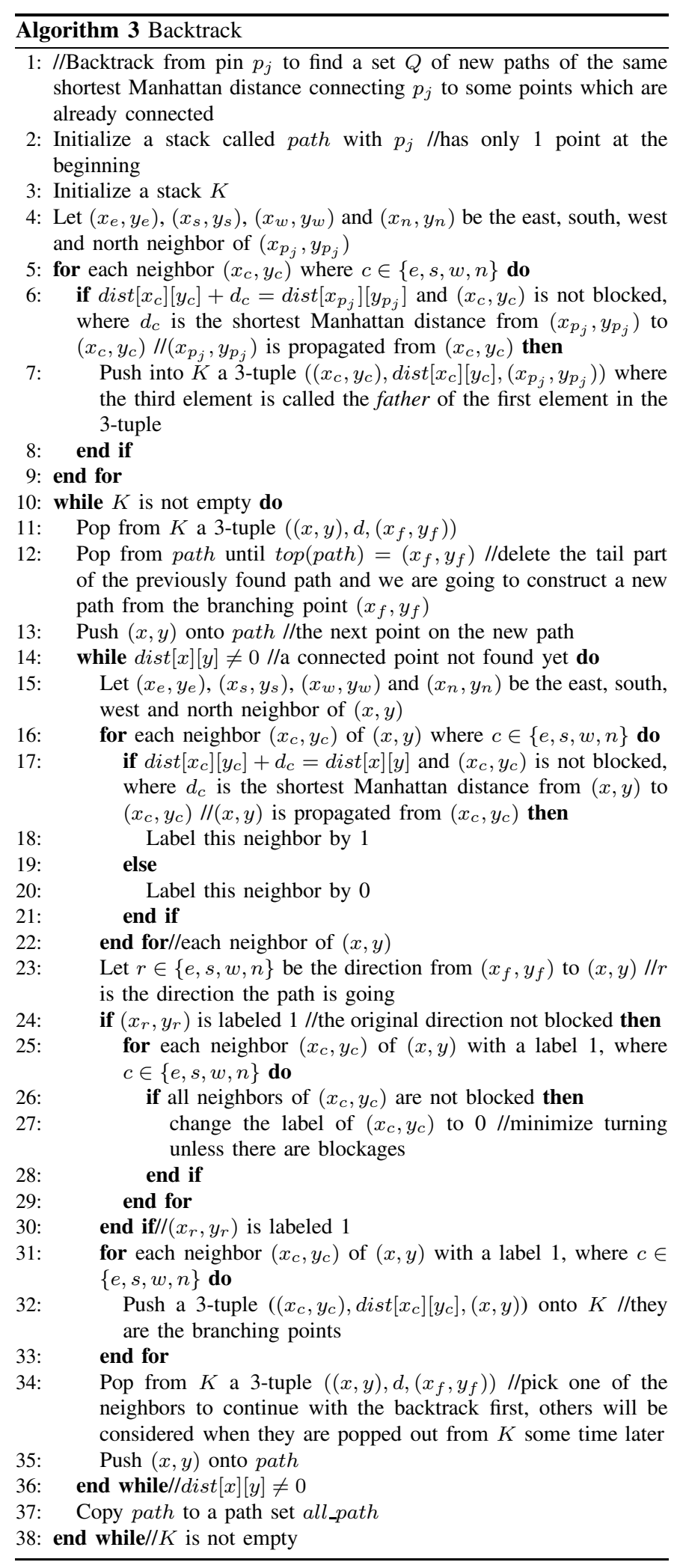

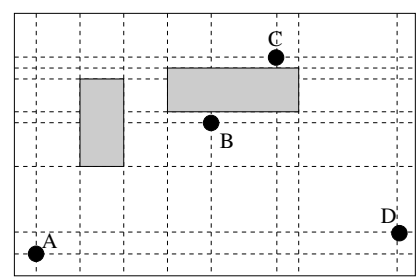

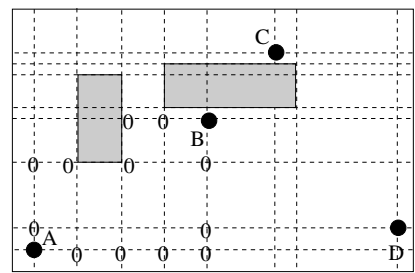

(c) All the points on the two paths found are set to zero. Continue propagation.

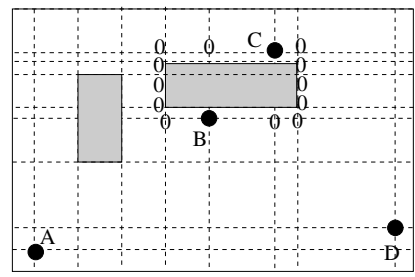

(e) All the points on the new paths found are set to zero. Continue propagation. (a) Start propagation from A.

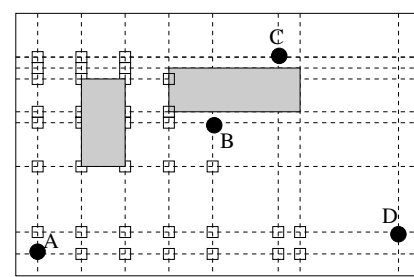

(b) Reach B at a distance 14. Other points at distance not more than 14 may also be reached.

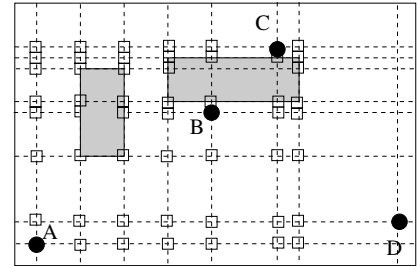

(d) Reach $\mathrm{C}$ at an additional distance of 8 .

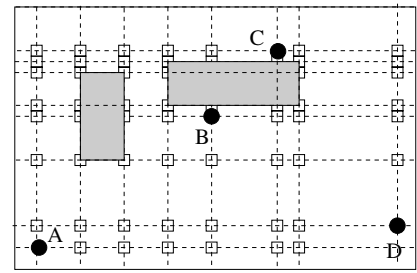

(f) Finally, all the pins are reached
Fig. 2. An example illustrating our propagation step

stored in $K$ are 3-tuples $\left((x, y), d,\left(x_{f}, y_{f}\right)\right)$ meaning that this step is going from $\left(x_{f}, y_{f}\right)$ to $(x, y)$ and the shortest distance of $(x, y)$ from a connected point is $d$, i.e., $\operatorname{dist}[x][y]=d$. From each branching point, a feasible path will be constructed step by step until reaching a point which is already connected, i.e., dist [ ][ ] $=0$ (line 14 to 36). A variable path is used to remember the current path under construction. On line 12 , it is popped until getting to $\left(x_{f}, y_{f}\right)$ which is a branching point, and we are going to construct another feasible path starting from that point. In the while-loop that computes a feasible path (line 14 - 36), a variable label is used to remember whether a point is a branching point and to be stored in $K$. We will try to move in the same direction without bending if no blockages are seen (line 26 to 28). If there are blockages, we will find all possible paths moving around the blockages. After a new path is found, it will be stored in the path set all_path. At the end, all feasible paths of the same shortest Manhattan distance will be stored in all_path.

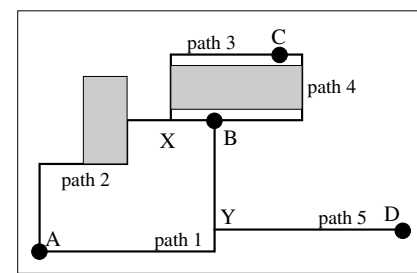

Fig. 3. A number of paths moving around the obstacles will be found. 


\section{Finding MST}

After finding a set of all paths, we can look at the ending points of each path. Since they are either a pin or a Steiner point, we can identify the positions of all the Steiner points immediately after this step. Then, we need to find the path lengths between the set of pins and Steiner points if they are connected. These distances can be computed very efficiently by, again, tracing each path once. When we are tracing a path $p$, we can identify the positions of the Steiner points or the pin points, and we can tell the distances between them easily. A Steiner tree $T$ will be constructed based on these distances and paths to connect all the points. It may happen that in $T$, some paths are just connected to some Steiner points only. We can remove these edges by removing recursively those leaf nodes which are Steiner points. After this, the tree give a reasonable route connecting all the pins and avoiding the obstacles. An example of this MST construction step is shown in Fig. 4.
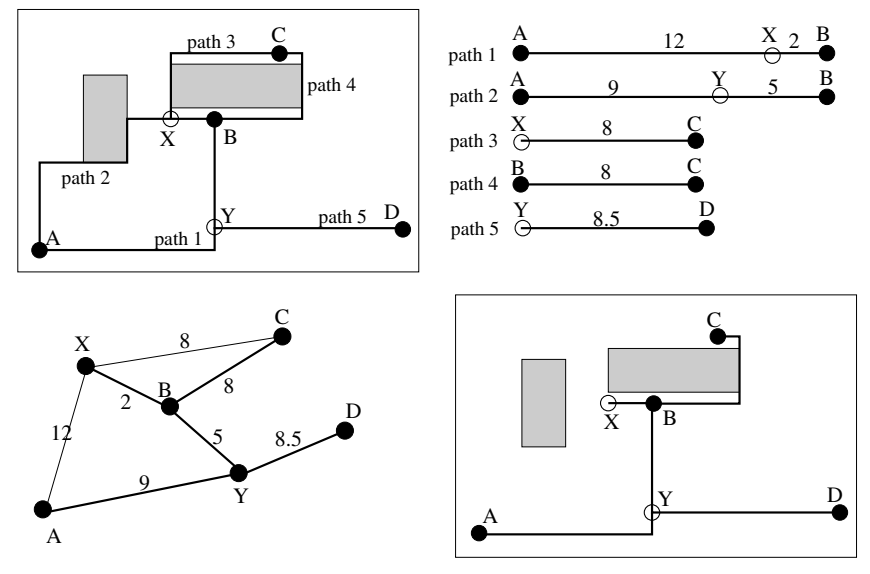

Find a MST between the points.

Route obtained from the MST.

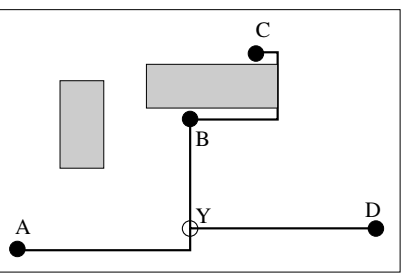

Remove dangling connection.

Fig. 4. An example illustrating how we find the Steiner points and path lengths

\section{E. Local Refinement Scheme}

Based on the results generated by the multi-pin maze routing engine, we will perform a post-processing step to further reduce the wire length. In this post-processing step, we will first identify all the pin points, Steiner points and turning points from the paths we computed. The original path information can be easily transformed to these point to point neighboring information by tracing each path once. After transformation from the path information to the point to point information, we will perform our local refinement scheme. In this local refinement scheme, for each point, we will first check whether it forms one of the topologies shown in Fig. 5. If such topologies exist, we will try to form a cycle by adding one straight edge (dotted line) between the two parallel paths. A longest path on this cycle formed will then be deleted to reduce the total wire length.
We will consider in details all the possible cases of having pins or Steiner points lying on this cycle. This local refinement scheme is more general than the U-shape pattern refinement in [15]

\section{EXPERIMENTAL RESULTS}

We implemented our algorithm in the $\mathrm{C}$ programming language and all the experiments were performed in a computer with a $2.2 \mathrm{GHz}$ Intel Pentium processor and 4GB memory running in the Linux environment. There are 22 benchmark circuits, five industrial test cases (ind1-ind5) from Synopsys, twelve test cases used in [12] (rc1rc12), and five randomly generated test cases from [15]. We compared our algorithm with the approach in [15], which gives the most updated results for this problem. The results of [15] shown below are directly cited from [15] and their experiments were performed on a $2 \mathrm{GHz}$ AMD-64 machine with $8 \mathrm{~GB}$ memory under the Ubuntu 6.06 operation system.

Table I lists the total wire length of the Steiner tree constructed by the two algorithms. We can see from the eighth column that without the post processing step we can improve over [15] by $1.73 \%$ on average. After applying the post processing step, we can improve over [15] by $2.01 \%$ on average. Since the solutions obtained might already be very close to the optimal, it is more meaningful to compare the differences from the optimal. The fourth column of Table I shows the edge length of the rectilinear Steiner minimal tree of all the pins without considering the blockages, which is a lower bound to the optimal solution. We can see that the improvement over the differences from this lower bound of the optimal solution is $27.04 \%$, and we can imagine that the improvement over the differences from the optimal solution will be even larger. Table II compares the CPU time of the two approaches. From this table, we can see that our method is similar to [15] in terms of running time and is sufficiently efficient. For the test case of rc12, there are many blockages and the pins' positions are evenly distributed in the 2-dimensional space, so the running time is longer than that of the other test cases.

\begin{tabular}{|c|c|c|c|c|c|}
\hline Test Cases & \multicolumn{2}{|c|}{ CPU Time (second) } & Test Cases & \multicolumn{2}{c|}{ CPU Time (second) } \\
\cline { 2 - 3 } & {$[15]$} & Ours & & {$[15]$} & Ours \\
\hline ind1 & $<0.01$ & $<0.01$ & rc7 & 0.43 & 0.65 \\
ind2 & $<0.01$ & 0.04 & rc8 & 0.83 & 1.18 \\
ind3 & $<0.01$ & $<0.01$ & rc9 & 0.91 & 1.49 \\
ind4 & $<0.01$ & $<0.01$ & rc10 & 0.62 & 0.26 \\
ind5 & $<0.01$ & $<0.01$ & rc11 & 3.15 & 0.76 \\
rc1 & $<0.01$ & 0.05 & rc12 & 118.52 & 147.14 \\
rc2 & $<0.01$ & 0.10 & rt1 & 0.06 & 0.16 \\
rc3 & $<0.01$ & 0.09 & rt2 & 0.11 & 0.55 \\
rc4 & $<0.01$ & 0.11 & rt3 & 0.47 & 0.20 \\
rc5 & 0.01 & 0.08 & rt4 & 0.95 & 0.33 \\
rc6 & 0.24 & 0.56 & rt5 & 2.06 & 2.78 \\
\hline
\end{tabular}

TABLE II

COMPARISONS ON THE CPU TIME.

\section{CONCLUSION}

We have proposed a new heuristic method to construct obstacleavoiding rectilinear Steiner minimum trees (OARSMT). Experimental results show that our approach is effective and efficient and it outperforms the best previous approach on this problem.

\section{REFERENCES}

[1] K.L. Clarkson and S. Kapoor and P.M. Vaidya, "Rectilinear Shortest Paths through Polygonal Obstacles in $O\left(n \log ^{2} n\right)$ ", Proceedings ACM Symposium on Computational Geometry, pp.251-257, 1987. 


\begin{tabular}{|c|c|c|c|c|c|c|c|c|c|}
\hline \multirow[t]{2}{*}{ Test Cases } & \multirow[t]{2}{*}{$m$} & \multirow[t]{2}{*}{$k$} & \multirow[t]{2}{*}{ GeoSteiner $(A)$} & \multicolumn{3}{|c|}{ Total Edge-Length } & \multicolumn{3}{|c|}{ Improvement $(\%)$} \\
\hline & & & & {$[15](B)$} & Ours $(C)$ & Ours $(D)$ & $(B-C) / B$ & $(B-D) / B$ & $(B-D) /(B-A)$ \\
\hline ind1 & 10 & 32 & 604 & 632 & 619 & 619 & 2.06 & 2.06 & 46.43 \\
\hline ind 2 & 10 & 43 & 9100 & 9600 & 9500 & 9500 & 1.04 & 1.04 & 20.00 \\
\hline ind 3 & 10 & 50 & 587 & 613 & 600 & 600 & 2.12 & 2.12 & 50.00 \\
\hline ind 4 & 25 & 79 & 1078 & 1121 & 1100 & 1096 & 1.87 & 2.23 & 58.14 \\
\hline ind5 & 33 & 71 & 1295 & 1364 & 1367 & 1360 & -0.22 & 0.29 & 5.80 \\
\hline $\mathrm{rc1}$ & 10 & 10 & 25290 & 26900 & 25980 & 25980 & 3.42 & 3.42 & 57.14 \\
\hline $\mathrm{rc} 2$ & 30 & 10 & 39170 & 42210 & 42280 & 42010 & -0.17 & 0.47 & 6.58 \\
\hline $\mathrm{rc} 3$ & 50 & 10 & 51900 & 55750 & 54560 & 54390 & 2.13 & 2.44 & 35.32 \\
\hline re4 & 70 & 10 & 54910 & 60350 & 59740 & 59740 & 1.01 & 1.01 & 11.21 \\
\hline rc5 & 100 & 10 & 71260 & 76330 & 75040 & 74650 & 1.69 & 2.20 & 33.14 \\
\hline rc6 & 100 & 500 & 76356 & 83365 & 81768 & 81607 & 1.92 & 2.11 & 25.08 \\
\hline $\mathrm{rc} 7$ & 200 & 500 & 105003 & 113260 & 111592 & 111542 & 1.47 & 1.52 & 20.81 \\
\hline $\mathrm{rc} 8$ & 200 & 800 & 107416 & 118747 & 116168 & 115931 & 2.17 & 2.37 & 24.85 \\
\hline $\mathrm{rc} 9$ & 200 & 1000 & 105698 & 116168 & 113642 & 113460 & 2.17 & 2.33 & 25.86 \\
\hline $\mathrm{rc} 10$ & 500 & 100 & 161790 & 170690 & 168520 & 167620 & 1.27 & 1.80 & 34.49 \\
\hline $\mathrm{rc} 11$ & 1000 & 100 & - & 236615 & 236359 & 235283 & 0.11 & 0.56 & - \\
\hline $\mathrm{rc} 12$ & 1000 & 10000 & - & 789097 & 765541 & 761606 & 2.99 & 3.48 & - \\
\hline rt1 & 10 & 500 & 1817 & 2267 & 2231 & 2231 & 1.59 & 1.59 & 8.00 \\
\hline $\mathrm{rt} 2$ & 50 & 500 & 44214 & 48441 & 47730 & 47297 & 1.47 & 2.36 & 27.06 \\
\hline $\mathrm{rt3}$ & 100 & 500 & 7579 & 8368 & 8222 & 8187 & 1.74 & 2.16 & 22.94 \\
\hline $\mathrm{rt} 4$ & 100 & 1000 & 7634 & 10306 & 9917 & 9914 & 3.77 & 3.80 & 14.67 \\
\hline $\mathrm{rt5}$ & 200 & 2000 & 42608 & 53993 & 52664 & 52473 & 2.46 & 2.82 & 13.35 \\
\hline Average & & - & - & - & - & - & 1.73 & 2.01 & 27.04 \\
\hline
\end{tabular}

blockages. $C$ is the edge length before applying the local refinement scheme and $D$ is the edge length after applying the local refinement scheme.

TABLE I

COMPARISON ON THE TOTAL EDGE-LENGTH.
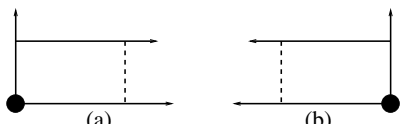

(b)

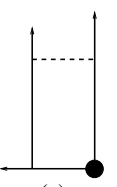

(e)

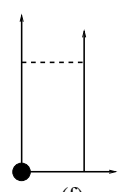

(f)

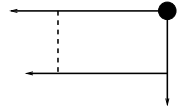

(c)

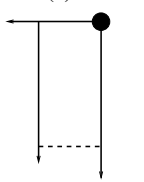

(g)

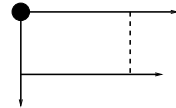

(d)

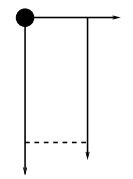

(h)
Dotted edges are the paths we consider adding to form a cycle

Fig. 5. Local Refinement Scheme

[2] Y.F. Wu and P. Widmayer, M.D.F. Schlag and C.K. Wong, "Rectilinear Shortest Paths and Minimum Spanning Trees in the Presence of Rectilinear Obstacles", IEEE Transaction on Computer-Aided Design, Vol.36, No.3, pp.321-331, 1987.

[3] J. Ganley and J.P. Cohoon, "Routing a Multi-terminal Critical Net: Steiner Tree Construction in the Presence of Obstacles", Proceedings International Symposium on Circuits and Systems, pp.113-116, 1994.

[4] S.Q. Zheng and J.S. Lim and S.S. Iyengar, "Finding Obstacleavoiding Shortest Paths using Implicit Connection Graphs", IEEE Transaction on Computer-Aided Design, Vol.15, No.1, pp.103-110, 1996.

[5] Y. Yang and Q. Zhu and T. Jing and X. Hong and Y. Wang, "Rectilinear Steiner Minimal Tree Among Obstacles", Proceedings IEEE ASICCON, pp.348-351, 2003.

[6] H. Wu and Z. Feng and T. Jing and X. Hong and Y. Yang and G. Yu and X. Hu and G. Yan, "FORst: a 3-step Heuristic for Obstacleavoiding Rectilinear Steiner Minimal Tree Construction", Journal of Information and Computational Science, pp.107-116, 2004.

[7] T. Lengauer, "Combinatorial Algorithms for Integrated Circuit Layout", Wiley, England, 1990.
[8] H. Wu and T. Jing and X. Hong and Z. Feng and X. Hu and G. Yan, "An-OARSMan: Obstacle-avoiding Routing Tree Construction with Good Length Performance", Proceedings ASP-DAC, pp.7-12, 2005.

[9] Z. Shen and C. Chu and Y. Li, "Efficient Rectilinear Steiner Tree Construction with Rectilinear Blockages", Proceedings ICCD, pp.38$44,2005$.

[10] H. Zhou, "Efficient Steiner Tree Construction Based on Spanning Graphs", IEEE Transactions on Computer-Aided Design, Vol.23, No.5, pp.704-710, 2004.

[11] Y. Shi and T. Jing and L. He and Z. Feng and X. Hong, "CDCTree: Novel Obstacle-avoiding Routing Tree Construction based on Current Driven Circuit Model", Proceedings ASP-DAC, 2006.

[12] Z. Feng and Y. Hu and T. Jing and X. Hong and X. Hu and G. Yan, "An $O(n \operatorname{logn})$ Algorithm for Obstacle-avoiding Routing Tree Construction in the $\lambda$-geometry Plane", Proceedings ISPD, pp.48-55, 2006.

[13] P.C. Wu and J.R. Gao and T.C. Wang, "A Fast and Stable Algorithm for Obstacle-avoiding Rectilinear Steiner Minimal Tree Construction", Proceedings ASP-DAC, pp.262-267, 2007.

[14] R. Hentschke and J. Narasimham and M. Johann and R. Reis, "Maze Routing Steiner Trees with Effective Critical Sink Optimization", Proceedings ISPD, 2007.

[15] C.W. Lin and S.Y. Chen and C.F. Li and Y.W. Chang and C.L. Yang, "Efficient Obstacle-avoiding Rectilinear Steiner Tree Construction", Proceedings ISPD, 2007. 